\title{
"Riesgo", "peligrosidad" e "implicacion subjetiva”: un analisis de las decisiones de internacion psiquiatrica en la ciudad de Buenos Aires*
}

María Jimena Mantilla ${ }^{1}$

MANTILLA, M.J. "Risk", "dangerousness" and "subjective implication": an analysis from psychiatric hospitalization decisions in the city of Buenos Aires. Interface - Comunic., Saude, Educ., v.14, n.32, p.115-26, jan./mar. 2010.

This paper describes and analyzes the decision-making process for psychiatric hospitalization using an ethnographic approach. It concentrates on the issue of dangerousness and risk, which are the conditions that define hospitalization criteria from a psychiatric perspective. It analyzes the psychoanalytical arguments regarding hospitalization as a therapeutic strategy. The results from the analysis show that decision on whether to hospitalize an individual are made through the practical, situational and contextual knowledge presented on each occasion. The professional's institutional position and membership of a specialty, the theoretical readings of the patient's situation and, finally, the availability of alternative resources are factors involved in such decisions.

Keywords: Hospitalization. Dangerousness. Psychiatry. Psychoanalysis.
O artigo descreve e analisa os processos de decisão de hospitalização psiquiátrica a partir de uma aproximação etnográfica. Concentra-se na questão da periculosidade e do risco, condições que definem o critério de internação desde uma perspectiva psiquiátrica. Analisa os argumentos psicanalíticos sobre as hospitalizações como estratégia terapêutica. Os resultados da análise mostram que internar ou não internar a uma pessoa é decidido mediante um saber prático, situacional e contextual posto em jogo em cada momento. Nessa decisão operam o lugar institucional do professional, a pertencimento a uma especialidade, as leituras teóricas sobre a situação do paciente e finalmente, a disponibilidade de recursos alternativos.

Palavras-chave: Hospitalização.

Periculosidade. Psiquiatria. Psicanálises.
*Este artículo es producto del análisis de los resultados de mi tesis de maestría "Prácticas y discursos "psi" en torno a las internaciones. Etnografía de un hospital psiquiátrico de la ciudad de Buenos Aires. ${ }^{1}$ Maestría en investigación en Ciencias Sociales. Universidad de Buenos Aires, Argentina. Instituto de Investigaciones Gino Germani, Comisión Nacional de Investigaciones Científicas y Tecnológicas. Calle Penna, 705, $1^{\circ} \mathrm{C}$ Vicente López. Buenos Aires, Argentina. jimenamantilla@yahoo. com.ar 


\section{Introducción}

En la actualidad el sistema de salud mental argentino se caracteriza por ser básicamente asilar, la internación psiquiátrica constituye una de las pocas herramientas terapéuticas para aliviar las situaciones de padecimiento mental. La demanda de internación supera en algunos casos la capacidad de los servicios. No obstante, en las últimas décadas asistimos a un progresivo cuestionamiento de la institución manicomial impulsado por debates legislativos, proyectos políticos de reforma psiquiátrica y experiencias desmanicomializadoras concretas en distintas partes del país. En este contexto, resulta necesario examinar no sólo las condiciones sociales, económicas y culturales que favorecen la ocurrencia de las internaciones psiquiátricas sino también las modalidades, los actores y los criterios que conforman los procesos de decisión/clasificación que anteceden a las internaciones psiquiátricas.

En el campo de la psicopatología las investigaciones buscan establecer relaciones estadísticas entre la internación psiquiátrica, las características sociodemográficas, el diagnóstico de los pacientes y el grado de peligrosidad (Glover, Arts, Suresh, 2006; Folino, Franklin 2004; Rabinowitz, Massad, Fennig, 1995; Slagg, 1993; Somoza, Somoza, 1993; Appelbaum, Hamm, 1982). Los aspectos vinculados con los criterios de decisión son poco estudiados. En este sentido, las internaciones psiquiátricas son usualmente investigadas desde la epidemiología psiquiátrica. Estas investigaciones hacen una discriminación por diagnóstico psiquiátrico y deterioro cognitivo y social como variables centrales para explicar las causas de múltiples internaciones en la trayectoria de un mismo paciente (Strejilevich, 2001). La mayoría de los trabajos en esta línea intenta determinar factores predictivos de rehospitalización. (Gunnel et al., 2008; Bindman, Reid, Szmukler, 2005; Bergen, 1998; Daniels, 1998; Appleby, 1996; Figuerido, 1995; Laessle, 1987). Otras investigaciones expresan que el número de reingresos ha ido aumentando en los últimos años y que el costo de las reinternaciones es un factor central para que el tema se instale en la investigación (Agrest et al., 1993). Los trabajos mencionados, sin embargo, no indagan en las bases en que se apoyan las decisiones de internación. Asimismo, en los abordajes de las ciencias sociales acerca de la problemática de la salud mental, estos aspectos son poco explorados. Para comprender cómo se estructuran - en base a que tipo de indicadores y criterios - las decisiones de internación, se requiere un abordaje etnográfico que permita documentar in situ dichos procesos.

El objetivo de este artículo es examinar las decisiones de internación psiquiátrica a partir de cómo los saberes psiquiátricos y psicoanalíticos interpretan y definen las nociones de "peligrosidad", "riesgo" e "implicación" o "responsabilidad subjetiva". En primer lugar describo la noción de peligrosidad, condición médica-legal de internación psiquiátrica. Analizo el concepto de "riesgo para sí y para terceros", criterio a través del cual se evalúa la peligrosidad. Asimismo planteo como tensión inherente al análisis de la peligrosidad, la posibilidad de sobreestimar o subestimar la ocurrencia del riesgo para sí o para terceros. En segundo lugar, examino los argumentos psicoanalíticos acerca de la pertinencia y el significado de la internación más allá del criterio de peligrosidad.

A lo largo de estas páginas muestro cómo las decisiones de internación se producen a partir de una serie de factores que no responden necesariamente a los discursos y prácticas "psi" en tanto saberes objetivos. Internar o no internar se resuelve a través de un saber práctico, situacional y contextual que se pone en juego en cada momento.

\section{Notas metodológicas}

Este trabajo se inscribe en un proyecto de investigación más amplio que tiene por objetivo analizar los discursos y prácticas en torno a las internaciones psiquiátricas desde una perspectiva etnográfica en un hospital de emergencias psiquiátricas. Ajustándose a los criterios metodológicos cualitativos, he realizado entrevistas en profundidad, observación participante, y conversaciones informales con los pacientes, profesionales y familiares.

La mayoría del material presentado en este artículo proviene de observaciones en la guardia del hospital y entrevistas en profundidad realizadas a profesionales psiquiatras y psicólogos de distintos sectores del hospital. 


\section{El discurso de la peligrosidad y la internación psiquiátrica}

En Argentina la noción de peligrosidad constituye el criterio médico-legal de internación psiquiátrica. La noción de peligrosidad nace de la mano de la teoría penal y su origen se remonta al siglo XIX, momento en el cual se conforma el modelo asilar que sustenta la institución psiquiátrica tradicional (Foucault, 2000). La asociación entre locura y peligrosidad instaura la superposición entre punición y tratamiento, tutela y locura, dando por resultado la restricción de derechos y deberes y la vinculación, así más no sea implícita, con las conductas delictivas.

El concepto de peligrosidad se articula también con el modelo correccional propuesto por el alienismo entre fines del siglo XIX y comienzos del siglo XX. EI modelo correccional se conforma por instituciones de secuestro cuyo objetivo es propiciar la defensa social (Murillo, 1999). La noción de corrección se liga con un estado peligroso que es preciso modificar a través de dispositivos de encierro como el manicomio y la cárcel. Por ello, la evaluación de la peligrosidad social se convierte en un instrumento que pretende establecer los riesgos y diagnosticar las virtualidades del comportamiento.

La internación psiquiátrica adquiere sentido en este paradigma y la función de la psiquiatría es delimitar el grado de peligrosidad de un individuo y evitar un retorno prematuro a la sociedad cuando sea susceptible de poner en peligro a uno de sus miembros.

En esta dinámica, la noción de peligrosidad articula el discurso psiquiátrico, el jurídico y el institucional. Se convierte en un término polisémico que si bien remite a los orígenes de la psiquiatría aún funciona en la actualidad como criterio de internación psiquiátrica, pese a las controversias y dificultades en su definición.

En términos generales, los profesionales acuerdan en considerar necesaria una internación siempre y cuando "haya peligrosidad", la dificultad radica en su definición. La noción de peligrosidad se enmarca en una tensión inherente a sí misma. Oscila entre el riesgo de sobreestimarla y subestimarla como un dilema imposible de evitar, no se trata de un error en su evaluación sino que forma parte de la imposibilidad de establecer un juicio libre de incertidumbre.

Actualmente en el campo de los derechos humanos y la salud mental se apunta a una revisión de la noción de peligrosidad acorde con las recomendaciones de la Organización Mundial de la Salud y la Organización de las Naciones Unidas². Los análisis críticos de la peligrosidad plantean dejar de considerarla como una propiedad individual de cada paciente para situarla en un conjunto complejo de relaciones (Diaz Usandivaras et al., 2001). Estas posturas críticas proponen invalidar la noción de "paciente peligroso" a fin de contemplar las situaciones puntuales de riesgo, respecto de quiénes y bajo qué circunstancias concretas pueden manifestarse conductas peligrosas. Asimismo, aún pese a una evaluación positiva de la peligrosidad, estos autores sugieren considerar otras opciones de tratamiento - control ambulatorio, hospital de día, cuidado familiar, etcétera - y no recurrir a la internación en todos los casos. Sin embargo, los estándares legales establecidos en el Código Civil respecto a la internación psiquiátrica son extremadamente generales y ambiguos, y permiten la detención de personas en instituciones psiquiátricas bajo una gama amplia de circunstancias.

Más allá de experiencias desmanicomializadoras concretas a lo largo de todo el país, no existen planes de salud mental a nivel nacional. Tal como afirman Larrobla y Botega (2000) en su investigación acerca de la situación de la salud mental en América del Sur, un dato que caracteriza a la Argentina es la desigualdad en materia de políticas y programas de atención en las diferentes jurisdicciones, como también la escasez de datos estadísticos y epidemiológicos. 
Asimismo, las consecuencias de la última dictadura militar son referidos como uno de los obstáculos para la transformación del sistema manicomial. A partir de la década del 50' hasta el golpe militar del 76' un grupo de psiquiatras influenciados por el psicoanálisis y el movimiento de salud mental y soportados tanto por gobiernos militares como civiles comenzaron un proceso de transformación de la psiquiatría con la intención de reemplazar los manicomios por salas de internaciones en hospitales generales, hospitales de día, comunidades terapéuticas, entre otros dispositivos (Carpintero, Vainer, 2005; Lakoff, 2005; Ablard, 2003). La interrupción de las prácticas innovadoras durante la dictadura, pese a la restauración democrática del 83' y las recomendaciones de los organismos internacionales, es aún un objetivo pendiente.

Ahora bien, la peligrosidad se inscribe en una noción de riesgo para sí o para terceros, el paciente es susceptible de hacerse daño a sí mismo o de hacerle daño a otro: "una autoagresión o heteroagresión". En general refiere a un daño físico, aunque también se trata de daños motivados por el comportamiento impropio del paciente. Las nociones de peligrosidad y riesgo se convierten en categorías que estigmatizan a los pacientes psiquiátricos en tanto refuerzan la asociación - poco comprobada - entre la enfermedad mental y la violencia.

Llevado al campo "psi", el análisis del riesgo obliga a una constante evaluación de las acciones e intenciones de los pacientes. La prevención de un comportamiento riesgoso constituye un elemento ineludible de la justificación de una internación. Se trata de un análisis especulativo en tanto responde a la virtualidad del comportamiento, más allá de que se apoya en determinadas situaciones concretas. A su vez, la evaluación del riesgo procura ordenar en las confusas situaciones que traen los pacientes y sus familias a la guardia psiquiátrica, una suerte de distribución de las responsabilidades respecto de quienes generan situaciones de riesgo. ¿Es el paciente el responsable de sus propios actos riesgosos? ¿Es la familia la que impulsa conductas peligrosas? De una u otra forma, el dispositivo "psi", con el respaldo de los saberes que intervienen, sanciona y distribuye responsabilidades, expresadas - a veces como culpas hacia los pacientes cuando no cumplen con sus deberes y obligaciones en tanto enfermos (Parsons, 1984) o hacia la familia en tanto productora de enfermedad mental.

Considerar los diferentes riesgos, pero no de los pacientes, sino hacia los pacientes, es decir los riesgos que sufren los pacientes durante las internaciones: violencia y abusos sexuales, pérdida de personalidad, entre otros (Quirk, Lelliot, Seale, 2004; Goffman, 1984, Rosenhan, 1974) contribuiría, en la medida de lo posible, a evitar la internación psiquiátrica como estrategia de tratamiento.

\title{
Riesgo para sí
}

\begin{abstract}
"A veces es una cuestión de disminuir el riesgo en el cual se está exponiendo un paciente, sea un riesgo suicida, un riesgo más por las conductas... paranoides, entonces irá a ver a todos los juzgados, todos los lugares CGP, buscando hacerle juicio a todo el mundo, digamos, que es una situación de riesgo porque cada vez eso genera más tensión, más nerviosismo, más reacciones psicóticas. Y otras veces se trata de disminuir el riesgo suicida del paciente, quizás en internación no se termina de resolver, pero por lo menos permite que se pueda seguir de a poco con un tratamiento ambulatorio". (Psiquiatra residente)
\end{abstract}

¿Cuáles son las situaciones que se clasifican como de "riesgo para sí"? En primer lugar, los intentos de suicidio. Si una persona cometió un atentado contra su vida es casi evidencia suficiente para indicar una internación. Se plantea la siguiente hipótesis: si fue capaz de hacerlo una vez, es muy probable que lo vuelva a repetir. No obstante se evalúan determinadas condiciones: si la persona está deprimida, si posee contención familiar, si se encuentra bajo una descompensación psicótica, entre otros aspectos que influyen en la decisión.

Para establecer el riesgo de suicidio se utilizan diferentes instrumentos clínicos: el diagnóstico, si hubo o no intentos previos, los antecedentes familiares y la intensidad de la "ideación suicida" o las "ideas de muerte", como suelen llamarla. También existen tests y cuestionarios mediante los cuales se completa la evaluación psiquiátrica. La evaluación del riesgo suicida constituye un eje de la práctica 
psiquiátrica desde el cual se efectúan otro tipo de intervenciones más allá de la internación: el ajuste de la medicación, la periodicidad en las consultas, la indicación de control familiar, entre otras.

Pese a los intentos por perfeccionar los instrumentos clínicos, la evaluación del suicidio, se enfrenta a la imposibilidad de determinar a priori su ocurrencia. No obstante, la responsabilidad legal y la genuina preocupación por la vida de los pacientes presionan a los psiquiatras a llegar a una definición.

Además del riesgo suicida, existen otros factores que configuran el riesgo "para sí", uno de ellos son los estados depresivos severos. En especial, algunos pacientes que suspenden actividades básicas para la supervivencia como la ingestión de alimentos. En este sentido, el cuerpo interviene en la evaluación de la peligrosidad y el riesgo: la negación a comer, la sobreingesta de medicación (ingerir altas dosis de fármacos con la intención de quitarse la vida), los cortes (mutilaciones), dan cuenta de la relación estrecha entre cuerpo y riesgo. El riesgo cristalizado en el cuerpo constituye una señal de alarma. Librada de dobles sentidos - a diferencia de la perspectiva psicoanalítica - una sobreingesta o cualquier otro tipo de intento de autoagresión es interpretado a partir de su gravedad física. Es decir, de cuán cerca estuvo o no de ocasionar muerte. El indicador resultante dará la pauta de alarma para una internación.

Por último, los estados maníacos y paranoides constituyen también "riesgo para sí" en tanto en ese tipo de situaciones los pacientes son susceptibles de estropear sus vínculos, perder sus trabajos, posesiones, gastar dinero en forma desenfrenada a partir de interpretar la realidad en forma errónea. Muchas veces cuando las creencias de los pacientes apuntan a un daño severo que un familiar pretendería hacerles, suelen coincidir con relaciones con cierto nivel de hostilidad o dependencia aunque no respondan a la creencia del paciente. Estas vivencias cargadas de ansiedad, angustia y desesperación, los exponen al riesgo. En estos casos, la peligrosidad deja de constituirse en un riesgo físico para el paciente y se convierte en un riesgo moral. Se trata de prevenir las faltas o desviaciones respecto de determinado orden familiar, laboral, comunitario. Mediante la operación psiquiátrica de clasificación del riesgo, las dimensiones morales de la vida de los pacientes se reconstituyen médicamente. El paciente es juzgado en sus conductas traducidas en síntomas de enfermedad mental.

Este tipo de riesgo moral confluye con algunas representaciones sobre la locura que se deslizan en la práctica "psi". Las asociaciones con el exceso, la impredicibilidad de los comportamientos, la disrupción y el quiebre, entre otras. Mitos, realidades, miedos, responsabilidades profesionales, tal es la diversidad de aspectos que se ponen en juego a la hora de clasificar la peligrosidad.

\section{Riesgo para terceros}

Los procesos de categorización del "riesgo para terceros" implican la evaluación de conductas que atenten contra otras personas. En general se trata de conductas paranoides, ya que se considera que el paciente podría hacerle daño a quienes en su delirio son sus agresores, es decir se convierte en un paciente violento, y por ende peligroso.

Pese a la existencia de algunos hechos puntuales de violencia y de situaciones concretas de riesgo para terceros, identifico cierta predisposición "psi" a clasificar conductas de los pacientes como paranoides. Las observaciones etnográficas sugieren que ante cualquier señal de queja, crítica o conflicto con algún familiar, amigo o conocido, se produce la sospecha de que el paciente "está paranoide". Dicha clasificación es a veces apresurada si se tiene en cuenta el lugar desvalorizado que ocupa el paciente psiquiátrico en la dinámica de sus relaciones cercanas, y por consiguiente su lógica predisposición a una actitud de desconfianza hacia los demás. Las intervenciones "psi" actúan bajo una presunción de anormalidad que tiñe la mirada hacia la mayoría de las acciones de los pacientes. El caso de Silvia ilustra una experiencia de descrédito hacia el discurso del paciente.

Silvia, 40 años, casada con tres hijos, estuvo internadas en la sala de mujeres, con el diagnóstico presuntivo de trastorno bipolar. A causa de una crisis económica había perdido su casa y vivía junto con su esposo y sus hijos en la casa de sus suegros. Desde el inicio de la internación denunciaba escenas de violencia tanto de parte de su esposo como de su suegra. Aún pese a la frecuencia de las denuncias, el psiquiatra no las consideraba. Desde su mirada formaban parte de los síntomas 
paranoicos de Silvia, quien, en medio de su período maníaco - y como reacción frente a la decisión de internación - se mostraba querellante. Tuvo que pasar un tiempo para que se dé intervención al servicio de violencia familiar y solo ocurrió en tanto el esposo reconoció explícitamente haberle pegado alguna vez.

El caso de Silvia, da cuenta de la dificultad en reconocer que esos discursos "querellantes" y "paranoicos" tengan alguna conexión con situaciones reales de conflictos, violencias y abusos. En tanto la palabra del paciente psiquiátrico, por su condición de enfermo mental se encuentra cuestionada, la primera lectura "psi" es la de un síntoma delirante o al menos un signo patológico. Esto no implica que las internaciones - y otras decisiones terapéuticas - se decidan sólo a partir de esas impresiones como únicos elementos de juicio, sino que se trata de una "disposición a" que subyace a las decisiones. En todo caso, las interpretaciones terapéuticas oscilarán en considerar a los pacientes como más o menos delirantes en función de múltiples elementos.

Sin embargo, en múltiples historias de pacientes, el delirio y la realidad se entremezclan, mostrando de manera difusa la separación construida entre uno y otro.

\section{Entre subestimar y sobreestimar la peligrosidad}

La evaluación de la peligrosidad atraviesa las distintas instancias de tratamiento: hospital de día, ambulatorio, consultas a la guardia. Cuando los profesionales tienen dudas acerca de si el paciente está desorientado, descompensado, o si abandonó la medicación, se ven obligados a analizar la peligrosidad y a evaluar la pertinencia de una internación. Sin embargo, no siempre les resulta fácil definir el riesgo. Tal como señala Doménech (2006), la evaluación de la peligrosidad entraña en sí misma un doble riesgo: el de sobreestimarla y el de subestimarla.

Algunas decisiones de internación están más relacionadas con un temor del profesional a que ocurra algo en un futuro incierto que con una situación concreta de riesgo. Algunos profesionales reconocen que sus propios miedos se ven involucrados en las decisiones. Otros reconocen que no siempre tienen la suficiente aptitud para ocuparse de pacientes graves en el contexto de un tratamiento ambulatorio.

“[...] También creo que se interna por demás, en este hospital a veces se interna por demás para cuidarse uno porque es mas cómodo también para el profesional, no nos olvidemos de eso, es más seguro tener un paciente internado que bancarte la incertidumbre y la angustia". (Psicóloga residente)

La angustia, la seguridad que implica una internación, la preocupación diaria por la vida de un paciente, son algunas de las dimensiones que influyen en la sobreestimación de la peligrosidad. La responsabilidad por un suicidio (sea o no fundada) actúa como un criterio que subyace a la decisión de internar. La responsabilidad legal y el temor a los juicios de mala praxis, no surgen en forma directa. Por el contrario, el miedo a "que se me mate un paciente" se encuentra vinculado a una cuestión humanitaria propia del compromiso corporal y emocional que les genera el trabajo en un hospital psiquiátrico.

La siguiente cita es parte del relato de una psicóloga acerca de una paciente que había comenzado a atender en tratamiento ambulatorio. El comentario apunta a mirar críticamente hacia el tratamiento anterior de la paciente, donde se ve reflejada una actitud profesional proclive a la internación. A veces, en el contexto de respuestas posibles a los problemas de salud mental, la internación resulta ser la única alternativa en la medida que no se conciben otras estrategias terapéuticas.

\footnotetext{
"Hoy me cuenta que la semana pasada vino con el bolso armado para que la internaran porque cada vez que le decían que tenía ganas de matarse la internaban. No es que la paciente está pidiendo la internación, aunque tampoco fueron compulsivas, pero de algún modo se estableció algo que frente a que ella diga que se quiere matar la respuesta es la internación. Porque, realmente la internaban porque decía que se quería matar, era la única posibilidad de trabajar sobre esas ideas". (Psicóloga de planta)
} 
En la otra cara del asunto se encuentra la posibilidad de subestimar el riesgo. Es el caso de una paciente que en una oportunidad llegó al hospital diciendo que quería matarse, algo que si bien repetía de tanto en tanto no derivaba en ningún intento. El psiquiatra la atendía desde tiempo atrás y por eso no se sorprendió con este anuncio. Desde la perspectiva del médico, la situación formaba parte de un modo común de actuar de la paciente. Sin embargo, al día siguiente se tiró bajo las vías del tren.

¿Fue posible determinar a priori la ocurrencia de este hecho? No trato de profundizar un análisis sobre el suicidio sino de reflexionar sobre su articulación con la peligrosidad en tanto criterio de internación. Desde el campo de la salud mental se puede argüir infinidad de cuestiones que tal vez fallaron al interior del tratamiento: un inadecuado seguimiento psiquiátrico de la paciente, ausencia de control farmacológico, de contención psicológica, entre otros. Más allá de las prácticas terapéuticas insuficientes o inadecuadas, es la propia noción de peligrosidad la que plantea dificultades al momento de su evaluación. La oscilación entre exagerar y subestimar el riesgo se convierte una tensión insoslayable en tanto se trata de cálculos estimativos y no de certezas absolutas.

\section{La peligrosidad según quien la evalúe}

"Los criterios de internación también son muy al ojo de cada uno". (Psiquiatra de planta)

Una primera cuestión a tener en cuenta es cuál es la posición institucional del profesional que indica una internación. En el hospital son los profesionales que pertenecen al servicio de guardia quienes deciden formalmente la internación. Los profesionales de los otros servicios (consultorios externos, hospital de día, salas de internación) derivan a la guardia con indicación de internación, pero en última instancia la decisión pertenece a la guardia. Las observaciones en la guardia dan cuenta que la capacidad de decisión varía si se trata de un miembro de planta o residencia. Estos últimos, al ser profesionales en formación tienen menor capacidad de decisión. Hay que considerar que no siempre hay camas en el hospital (lugares para internar), por lo tanto no se interna por ausencia de disponibilidad de recursos, más allá de si el paciente tiene o no criterio de internación. En esos casos se deriva a otros nosocomios aún con el riesgo de que la situación sea similar. Es decir, los recursos informales, las estrategias de negociación y las condiciones institucionales forman parte de las contingencias de la decisión de internación.

Por otro lado, al examinar los procesos que anteceden a las internaciones es factible encontrar diferentes opiniones profesionales frente a un mismo paciente. En primer lugar, con los pacientes de consultorios externos que son derivados a la guardia con indicación de internación puede ocurrir que para el equipo tratante de consultorios externos, la decisión de internar derive de un proceso al que llegan convencidos. El problema se genera cuando acuden a la guardia junto con el paciente y se encuentra con dos tipos de obstáculos: o no hay camas (es decir no hay lugar para alojar al paciente, lo cual se convierte en un obstáculo institucional frecuente) o los psiquiatras de guardia no consideran que amerite una internación: "no tiene criterio de internación".

Frente a estas cuestiones, los profesionales de consultorios externos se ven envueltos en una actitud de preocupación constante, diversas idas y venidas a la guardia a fin de lograr que "le internen al paciente". Como consecuencia, la evaluación del riesgo se convierte en una actividad casi diaria, y al no lograr la internación se ven obligados a juzgar a cada momento las condiciones psicopatológicas del paciente. Como indican los datos etnográficos, el criterio de internación se modifica con el transcurso de las horas en tanto las evaluaciones "psi" e incluso el mismo paciente, cambian. Si el paciente está más tranquilo, el sufrimiento es menos intenso, o la ideación delirante se haya controlada se modifica el criterio de internación. En este punto, el hecho de considerar oportuna la internación de un paciente y no poder efectivizarla, muestra los aspectos relativos y cambiantes de dicha decisión. No se trata de ausencia de idoneidad profesional sino de los aspectos dinámicos de las situaciones de padecimiento en que se encuentran los pacientes y las formas en las que se comunican e interpretan dichos padecimientos en el marco de las relaciones terapéuticas. 
Respecto de las diferencias de criterios, los profesionales de consultorios externos afirman que es mayor el conocimiento que tienen de la historia del paciente en comparación con sus colegas de la guardia. Agregan que los profesionales de la guardia sólo observan una situación particular, un episodio tal vez no grave en sí mismo, pero sí en el contexto de una historia de vida.

Los distintos puntos de vista acerca de un mismo paciente entre la guardia y consultorios externos muestran al criterio de peligrosidad como una cuestión singular, vinculada - entre otros aspectos - a diferencias en cuanto a la interpretación del padecimiento del paciente.

En segundo lugar, también hay diferencias de opinión entre los profesionales de distintas disciplinas. Es decir, psiquiatra y psicólogo, como miembros del equipo tratante, pueden no coincidir en la clasificación de la peligrosidad y el criterio de internación. En esos casos se discute, se negocia, a veces se impone el criterio psiquiátrico en tanto se reconoce a la internación como un acto de mayor responsabilidad médica. Las situaciones no siempre son de riesgo evidente y admiten diferentes lecturas entre los miembros del equipo tratante.

En resumen, la evaluación de la peligrosidad es un proceso relativo al punto de vista de cada profesional, la disciplina a la que pertenece y la posición institucional que ocupa (es decir el servicio en el que desarrolla sus tareas y el nivel jerárquico de su posición).

\section{Matices al paradigma de la peligrosidad. El psicoanálisis y las decisiones} de internación

En esta sección examino los argumentos psicoanalíticos que los profesionales construyen acerca de la pertinencia y el significado de la internación en tanto estrategia terapéutica. Se trata de otras formas de entender la necesidad de internación, más allá del paradigma de la peligrosidad. En el contexto de tratamientos ambulatorios prolongados, los psicoanalistas afirman que la necesidad de internación surge como un momento en que es preciso establecer otro tipo de intervención. En estos casos, el criterio de internación se sustenta en una certeza producto del devenir del tratamiento.

La mayoría de los psicoanalistas pone el énfasis en la cuestión del "desenganche" del tratamiento ambulatorio como una causa que conduce a las internaciones. El objetivo de la internación apunta a "producir un movimiento subjetivo", modificar el lugar del sujeto en tanto lugar simbólico. Se trata de una noción en el discurso de los psicólogos a la que hacen referencia diariamente aludiendo a un supuesto significado común de la misma.

Asimismo, afirman que "el sujeto es responsable de su síntoma". Por ello, la alusión a la "responsabilidad subjetiva" orienta las decisiones de internación. Mediante ésta pretenden "instalar cierta inscripción de lo que la motivó, de la posición que el paciente tenía en la escena que provocó la internación". De este modo el paciente podría "modificar su posición". No obstante, en el caso que este tipo de implicación no se produce, es decir "el paciente no hace ningún movimiento", el psicoanalista puede interpretarlo como una falla en el tratamiento, producto de intervenciones inadecuadas o como una actitud poco favorable del paciente en tanto ausencia de disposición a "trabajar" 3.

En este proceso de búsqueda de implicación subjetiva, se desdibujan los escenarios sociales en los que ocurren las internaciones. Situaciones de violencia, de vulnerabilidad, de abandonos y carencias afectivas son el marco que precede a la internación. El llamado a la implicación subjetiva por fuera de determinismos socioculturales - o más bien más allá de los mismos - es propio de una concepción individualista del sujeto que subyace a los discursos "psi".

\footnotetext{
${ }^{3}$ La noción de "trabajar" alude al trabajo psíquico, el cual se motiva a partir de la técnica psicoanalítica de la asociación libre que insta al paciente a que se abandone a sus pensamientos espontáneos. En las situaciones etnográficas, los pacientes que producen recuerdos, asociaciones entre los hechos de su vida y la infancia, reflexiones en tanto a sus si mismos, son quienes se consideran "buenos pacientes". La noción de "trabajar" se asemeja en la perspectiva psiquiátrica con la del paciente que tiene "conciencia de enfermedad" y "adherencia al tratamiento".
} 
Con la internación buscan propiciar "una marca", un antes y después que permita "trabajar" con el paciente:

"Tal vez, con algunos pacientes, no con todos, la verdad es que no podría generalizarlo, con algunos pacientes me parece que se pudo como armar cierta inscripción distinta de lo que había motivado la internación. Como pasar de una pura actuación, o un puro así movimiento corporal de no entender porqué estaban internados y qué es lo que había pasado, como si se inscribiera cierta marca de lo que motivó la internación. Cierta marca de la posición que el paciente tenía en esa escena que motivó la internación o en esa coyuntura. Me parece que ahí se puede establecer como cierto corte". (Psicólogo de planta)

Durante el transcurso de la internación pretenden que el paciente "se apropie" de la misma, es decir que se involucre activamente con su internación, especialmente con las causas o hechos que antecedieron a la misma. Se trata de que "ubique las coordenadas de su descompensación" y la "posición que tenía en la escena de la internación".

La eficacia de las intervenciones es evaluada a partir de los dichos de los pacientes: "necesitaba un corte", "necesitaba parar", son interpretados como señales de eficacia terapéutica. No obstante, en este gesto se iguala la interpretación del paciente con la del psicoanalista. Los pacientes suelen reconocer que a veces durante la internación obtienen alivio de sus sufrimientos, sin embargo, ello no siempre supone una revisión de la "posición subjetiva".

Asimismo, desde la perspectiva psicoanalítica la interpretación del "riesgo para sí" difiere de la perspectiva psiquiátrica. Una sobreingesta de medicación no necesariamente implica criterio de internación. En todo caso, la gravedad del hecho va a depender del significado que adquiere a partir de comprender "a quién estuvo dirigida" la sobreingesta, el marco en el que se produce, el momento del tratamiento, entre otras preguntas a las que se pretende responder.

Como lo expresa la cita siguiente, existen al menos dos formas de interpretar el hecho. La primera es a partir del devenir del tratamiento, especialmente la relación terapéutica y el funcionamiento de la "transferencia". La segunda - más allá del tratamiento - mediante un análisis de "la dirección" del hecho. "Hacia quienes está dirigido" como metáfora de un pedido de ayuda, un reclamo, una muestra de desesperación, etc.

“[...] una paciente que atendí casi dos años en ambulatorio, mucho tiempo... dos años en tratamiento y acompañé la idea de una internación, sólo cuando pude concluir que se había roto el compromiso transferencial entre la paciente y yo, que algo de la confianza de la paciente hacia el tratamiento conmigo se había roto... por la vía de una sobreingesta. Pero te digo esto, no es que una sobreingesta me llevó a internarla, es que se venía hablando del tema, y fue una, una traición completa a lo que ella venía diciendo en transferencia conmigo. Con lo cual yo no tenía modo de pensar que nada de lo que ocurría en mi espacio podría ser una salvaguarda, un espacio de contención [...]

P: ¿Fue una sobreingesta con riesgo para sí, o...?

R: En ese momento no. Le hicieron un lavaje de estómago, pero no. había tomado todo un blister de risperidona, pero no es que se fuera a morir, era complicado que lo hubiera hecho, porque lo había hecho con el fin de matarse, no recuerdo si la dosis era letal o no...

$P$ : No, bueno, digo si era grave o...

R: Si fue, fue grave, pero por eso te digo, otras pacientes que en tratamiento se tomaron un blister de otra medicación o que se cortaron, qué se yo.. no, no, no digo yo se que, podía leer ahí un llamado, una... una actuación que admitía ciertas lecturas, que estaba dirigido, digo, había ciertos lazos que se preservaban, pese al riesgo que había implicado esa conducta [...] Ahora cuando esa paciente hizo esta sobreingesta y de algún modo se soltó del tratamiento, no se refirió más a mi, a mi herramienta, ella estaba en un cuadro muy depresivo, muy desesperanzada, digamos la desesperanza era en ella, no había nada que la pudiera ayudar, en ese punto si. Fue la primera internación". (Psicólogo residente, el énfasis es mío) 
Cómo expresa la cita, la sobreingesta se lee como una señal de que la paciente se desconectó del tratamiento. La confianza surge como un elemento clave del tratamiento, necesario para que éste tenga efectividad. Subyace a este pedido cierta exigencia de compromiso con la búsqueda de la verdad sobre el sujeto que ofrece el dispositivo psicoanalítico.

El contenido de las sesiones, lo que el paciente fue diciendo a lo largo del tiempo, los cambios en sus dichos, son interpretados no como metáforas de una situación de interacción asimétrica y sobre todo de construcción conjunta entre terapeuta y paciente sino como verdades ocultas que salen a la luz mediante el procedimiento analítico.

“[...] yo creo que lo veníamos, por eso te digo que, yo lo leí como una traición a sus propios dichos, un desconocimiento de los propios compromisos que ella había asumido, ¿no? esa libertad bajo palabra que uno instituye en un tratamiento, este "sos libre pero vamos a tener lo que usted diga" lo veníamos hablando, las últimas tres o cuatro entrevistas cuando ella estaba muy mal, se venía hablando del tema, como una posibilidad [...]

Fue una internación corta esa, creo que fue menos de un mes.

$P$ : ¿ la atendiste vos, en la internación?

R: No, yo decidí no volver a verla, esto se lo comuniqué, en función de lo que había pasado yo no iba a continuar el tratamiento [...]". (Psicólogo residente, el énfasis es mío)

Por otro lado, uno de los argumentos frecuentes que llevan a la decisión de internación es que el paciente con sus acciones "había traicionado con sus dichos el contrato de palabra que el tratamiento había establecido". La alusión al contrato de palabra como marco que regula la relación terapéutica es uno de los ejes de las intervenciones psicoanalíticas "nos vamos a atener a lo que usted dice". Cuando ese contrato se quiebra, se produce un cambio en la orientación del tratamiento. En algunos casos -según muestra la información etnográfica - ese cambio es vivido por el paciente como algo incomprensible que incluso le perjudica, ya sea por pasar de un tratamiento ambulatorio a una internación o por perder el vínculo con el terapeuta.

La "responsabilidad subjetiva" o los intentos por producir un "movimiento" o "una marca" mediante la internación, resumen la mirada psicoanalítica de las internaciones. La efectividad de estas pretensiones y lo asequible o no por los pacientes ameritan más de un interrogante. Al respecto son válidas las palabras de una psicóloga distante del psicoanálisis:

“Muchas veces en pacientes que están en externos, se dice que la internación es para hacer una marca, generar un corte y me parece que eso no es así, me parece mucho más auténtico decir: esto es un desborde hay que internarlo porque ya no sabemos que hacer. Si eso deja una marca lo sabremos después". (Psicóloga residente)

Por último, las lecturas psicoanalíticas acerca de la pertinencia de una internación se conjugan con las lecturas de la peligrosidad en una serie de prácticas e intervenciones que no hacen distinción entre psicoanalistas y psiquiatras. Por el contrario, se trata de lecturas que se dan entremezcladas y que se producen en situaciones y contextos específicos.

\section{Consideraciones finales}

En este artículo describí y analicé los criterios de internación psiquiátrica a partir de las perspectivas psiquiátricas y psicoanalíticas. Ya sea para lograr un cambio de posición subjetiva o como consecuencia de un análisis sobre la peligrosidad, la internación adquiere un sentido particular a los saberes que conforman el paradigma "psi". No obstante, como fue señalado, las decisiones de internación se producen a partir de una serie de factores que no responden necesariamente a los discursos y prácticas "psi" en tanto saberes objetivos. Internar o no internar se resuelve a través de un saber práctico, situacional y contextual que se pone en juego en cada momento, donde la posición institucional del 
profesional, la pertenencia a tal o cual disciplina, las lecturas teóricas sobre la situación del paciente, las interacciones con pacientes, familiares y otros actores y por último, la disponibilidad de recursos alternativos, se dan cita en una misma decisión.

El riesgo, la urgencia, el sufrimiento y la ausencia de recursos de integración social se encuentran en cada decisión de internación. Los profesionales "psi" se ven obligados a dar respuestas a situaciones que los exceden en su posibilidad de intervención y para las que cuentan con teorías y herramientas que no siempre son pertinentes.

Finalmente, a partir de los aspectos analizados surgen interrogantes para futuras investigaciones: ¿Cómo se diferencian y articulan las dimensiones del riesgo en un contexto social de vulnerabilidad y sufrimiento social como el actual?, es decir ¿cómo es posible establecer parámetros de riesgo cuando las situaciones de pacientes y familias son en general de incertidumbre, violencia y daño? Asimismo, ¿cuál será el lugar de la internación psiquiátrica, en un futuro contexto de reforma psiquiátrica?

\section{Referencias}

ABLARD, J. Authoritarianism, democracy and psychiatric reform in Argentina, 1943-83. Hist. Psychiatry, v.14, n.3, p.361-76, 2003.

AGREST, M. et al. Factores asociados a las reinternaciones de los pacientes psiquiátricos. Vértex Rev. Argentina Psiquiatr., v.5, p.167-75, 1993.

APPELBAUM, P.; HAMM, R. Decision to seek commitment: psychiatric decision making in a legal context. Arch. Gen. Psychiatry, v.31, p.355-62, 1982.

APPLEBY, L. et al. Lenght of stay and recidivism among patients with schizophrenia. Psychiatric Serv., v.47, n.9, p.985-90, 1996.

BERGEN, J. et al. Six month outcome following a relapse of schizophrenia. Aust. N. Z. Psychiatry, v.32, n.8, p.819-22, 1998.

BINDMAN, J.; REID, Y.; SZMUKLER, J. Perceived coercion at admission to psychiatric hospital and engagement with follow up. Soc. Psychiatr. Psychiatry Epidemiol., v.40, p160-6, 2005.

BONET, O. Saber e sentir: uma etnografia de aprendizagem da biomedicina. Rio de Janeiro: Fiocruz, 2004.

CARPINTERO, F.; VAINER, A. Las huellas de la memoria II: psicoanálisis y salud mental en la Argentina de los 60' y 70'. Buenos Aires: Tutopia, 2005.

DANIELS, B. et al. Predictability of rehospitalization over 5 years for schizophrenia, bipolar disorder and depression. Psychiatry, v.32, n.2, p.281-6, 1998.

DÍAZ USANDIVARAS, E.; JULIÁN, F. Enfermos y discapacitados mentales: apuntes médico-jurídicos sobre el concepto de peligrosidad. 2001. Disponible en: <http://www. psiconet.com/argentina/apsa2001/peligros.htm>. Acceso en: 17 jun. 2008.

DOMÉNECH, E. Peligrosidad: enigma o acertijo, s/f. 2006. Disponible en: <http:// www.selettigroup.com.ar>. Acceso en: 12 abr. 2007.

FOUCAULT, M. Historia de la locura en la época clásica. Bogotá: Fondo de Cultura Económica, 2000. v.1.

La verdad y las formas jurídicas. Barcelona: Gedisa, 1998.

FOLINO, O.; FRANKLIN, E. Nuevos aportes a la evaluación del riesgo de violencia. Med. Unab., v.7, n.20, p.99-105, 2004.

GOFFMAN, E. Internados: ensayos sobre la situación social de los enfermos mentales. Buenos Aires: Amorrurtu, 1984. 
GLOVER, G.; ARTS, G.; SURESH BABU, K. Crisis resolution/ home treatment teams and psychiatric admission rates in England. Br. J. Psychiatry, v.186, p.441-5, 2006.

GUNNEL, D. et al. Hospital admissions for self harm alter discharge from psychiatric inpatient care: cohort study. Br. Med. J., v.337, p.a2278, 2008. Disponible en: <http:// www.bmj.com/cgi/content/abstract/337/nov18_3/a2278>. Acceso en: 19 nov. 2009.

FIGUERIDO, J.L. et al. Predictors of rehospitalization in schizophrenia. Psychiatric Serv., v.46, n.11, p.1161-5, 1995.

LAESSLE, R. et al. Risk of rehospitalization of psychotic patients: a 6 years follow investigation using the survival approach. Psychopatol., v.20, n.1, p.48-60, 1987.

LAKOFF, A. Pharmaceutical reason: knowledge and value in global psychiatry. New York: Cambridge University Press, 2005.

LARROBLA, C.; BOTEGA, N.J. Las políticas de asistencia psiquiátrica y desinstitucionalización en América del Sur. Actas Esp. Psiquiátricas, v.28, n.1, p.22-30, 2000.

MURILLO, S. Gobernabilidad, locura y delito La mutación desde el modelo médicojuurídico al modelo tecnológico. Sudestada, v.1, p.12-23, 1999.

PARSONS, T. El sistema social. Madrid: Alianza Editorial, 1984.

QUIRK, A.; LELLIOT, P.; SEALE, C. Service user's strategies for managing risk in the volatile environment. Soc. Sci. Med., v.59, p.2573-83, 2004.

RABINOWITZ, J.; MASSAD, A.; FENNING, S. Factors influencing disposition decisions for patients seen in a psychiatric emergency service. Psychiatry Serv., v.46, p.712-8, 1995.

ROSENHAN, D. On being sane in insane places. Science, v.179, p.250-8, 1974.

SLAGG, N. Characteristics of emergency rooms patients that predict hospitalization or disposition to alterative treatments. Hosp. Community Psychiatry, v.44, p.252-6, 1993.

SOMOZA, E.; SOMOZA; J.R. A neutral network approach to predicting admission decisions in a psychiatric emergency room. Med. Decis. Making, v.13, p.273-80, 1993.

MANTILLA, M.J. "Riesgo", "peligrosidad" e "implicación subjetiva": un análisis de las decisiones de internación psiquiátrica en la ciudad de Buenos Aires. Interface - Comunic., Saude, Educ., v.14, n.32, p.115-26, jan./mar. 2010.

Este artículo describe y analiza los procesos de decisión de internación psiquiátrica a partir de una aproximación etnográfica. Se concentra en la cuestión de la peligrosidad y el riesgo; condiciones que definen el criterio de internación desde una perspectiva psiquiátrica. Analiza los argumentos psicoanalíticos sobre la hospitalización como estrategia terapéutica. Los resultados del análisis muestran que internar o no internar a una persona se decide mediante un saber práctico, situacional y contextual puesto en juego a cada momento. En esta decisión operan el lugar institucional del profesional, la pertenencia a una especialidad, las lecturas teóricas sobre la situación del paciente y la finalmente la disponibilidad de recursos alternativos.

Palabras clave: Hospitalización o internación psiquiátrica. Peligrosidad. Psiquiatría. Psicoanálisis. 